UCRL-JC- 127902

PREPRINT

\title{
Mapping and Inspection of Damage and Artifacts in Large-Scale Optics
}

\author{
F. Rainer
}

This paper was prepared for and presented at the 29th Annual Boulder Damage Symposium

Boulder, $\mathrm{CO}$

October 6-8, 1997

December 22, 1997

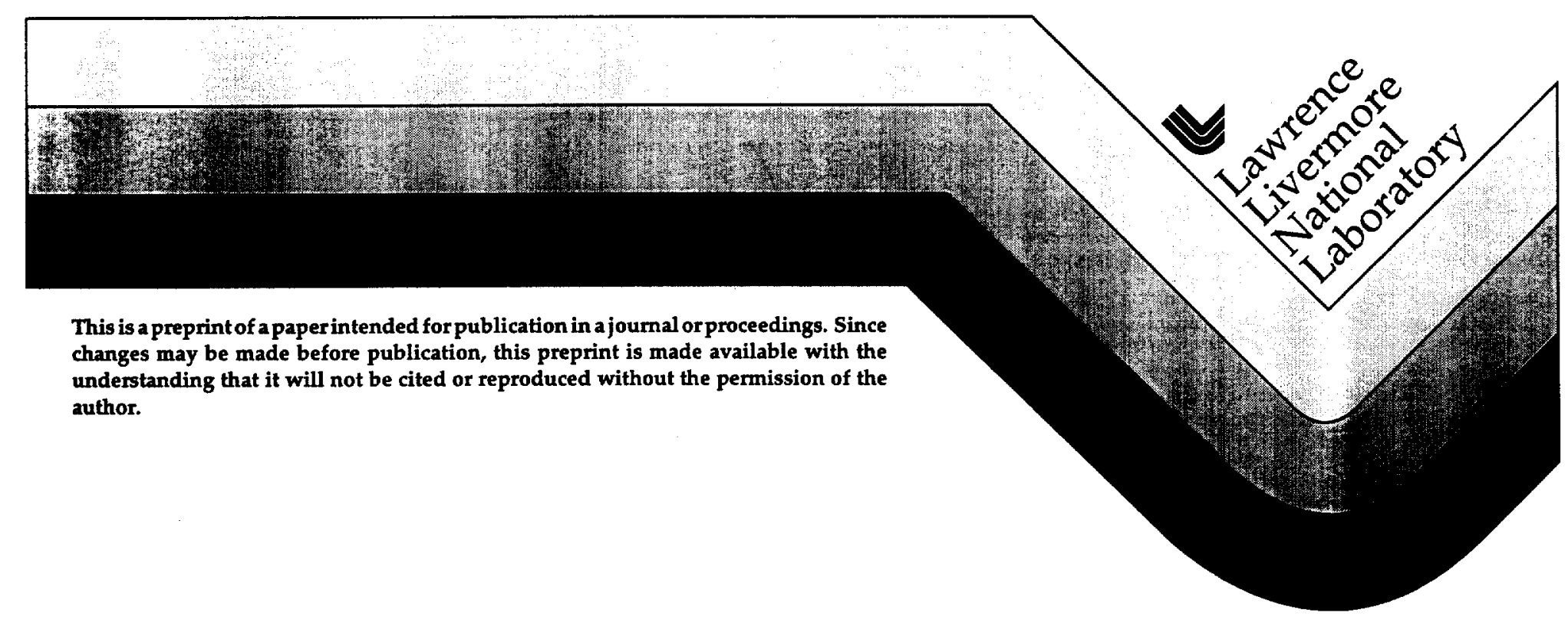




\section{DISCLAIMER}

This document was prepared as an account of work sponsored by an agency of the United States Government. Neither the United States Government nor the University of California nor any of their employees, makes any warranty, express or implied, or assumes any legal liability or responsibility for the accuracy, completeness, or usefulness of any information, apparatus, product, or process

disclosed, or represents that its use would not infringe privately owned rights. Reference herein to any specific commercial product, process, or service by trade name, trademark, manufacturer, or otherwise, does not necessarily constitute or imply its endorsement, recommendation, or favoring by the United States Government or the University of California. The views and opinions of authors expressed herein do not necessarily state or reflect those of the United States Government or the University of California, and shall not be used for advertising or product endorsement purposes. 


\title{
Mapping and inspection of damage and artifacts in large-scale optics
}

\author{
F. Rainer \\ Lawrence Livermore National Laboratory \\ P. O. Box 5508, L-487, Livermore, CA $94551-9900$
}

\begin{abstract}
We have developed tools and procedures for accurately and economically mapping and inspecting damage and artifacts in the bulk as well as on the surfaces of meter-sized optics intended for use on large-scale lasers such as Beamlet and NIF. Optics are illuminated by white light through the optic edge or externally at grazing incidence using linear fiber-optic arrays. A mega-pixel camera records a digital map of the entire optic with precise addresses of highlighted artifacts. From these coordinates, artifacts are located and digitally recorded with resolutions of less than $5 \mu \mathrm{m}$ using a long-working-distance microscope. Total internal reflection of edge illumination efficiently couples light into the entire optic to inspect for bulk artifacts such as bubbles, inclusions, edge-cladding flaws and laserinduced damage as well as surface scratches and pits which propagate into the substrate. Surface contamination such as dust, fingerprints, coating flaws and cleaning flaws are highlighted by external grazing illumination. The procedures permit accurate recording of the evolution of damage after many laser shots as well as correlation of damage from one optic to the next in a laser chain.
\end{abstract}

Keywords: damage, damage mapping, laser-induced damage, optics

\section{INTRODUCTION}

Optics being designed for use on large laser systems such as Beamlet and the National Ignition Facility (NIF) at the Lawrence Livermore National Laboratory (LLNL) are not only very large - approaching sizes of up to one meter - but also are being operated under high laser-fluence conditions. Even isolated defects in these large optics can lead to catastrophic damage unless the defects are either discovered prior to the optics being installed in the laser systems or at least monitored for potential growth during high-fluence operations. We have developed a costeffective, off-line diagnostic that can not only detect defects $\leq 5 \mu \mathrm{m}$ in size, but also can accurately pinpoint their location in three dimensions inside the bulk or on the surfaces of an optical substrate and quantify their morphology with high resolution digital images. This work represents a further refinement of on-line diagnostics employed for two years on Beamlet to monitor the evolution of damage of critical laser components.

As an example of the scale of the problem in finding potential problem sites, consider a typical optic with dimensions of $40 \times 40 \times 5 \mathrm{~cm}^{3}$. If this optic had 50 randomly distributed bulk defects on the order of $10 \mu \mathrm{m}$ in diameter, the total volume of the defects would be 12 orders of magnitude smaller than the optic volume. The defects might be found as enlarged damage sites after one or more full-area laser system shots or after several hours of raster scanning with a small laser beam. However, it would have been very unlikely that the cause of damage could have been definitively ascribable to pre-existing defects within the optic, laser-beam modulation from defects in upstream optics, or naturally occurring laser-beam hot spots. The isolated nucleation sites for potential damage in the first two cases would have been almost impossible to detect in such a large volume.

\section{DAMAGE MAPPING SYSTEM CONFIGURATION}

The damage mapping system consists of three components: (1) the optic under test held within a mounting frame, (2) a mega-pixel digital camera and illumination system to detect and quantify defect locations and (3) a long-working-distance microscope, three-dimensional orthogonal translation stages, and a back-lit illumination system to quantify the detailed morphology of the defects or damage. These are displayed in Fig. 1. 


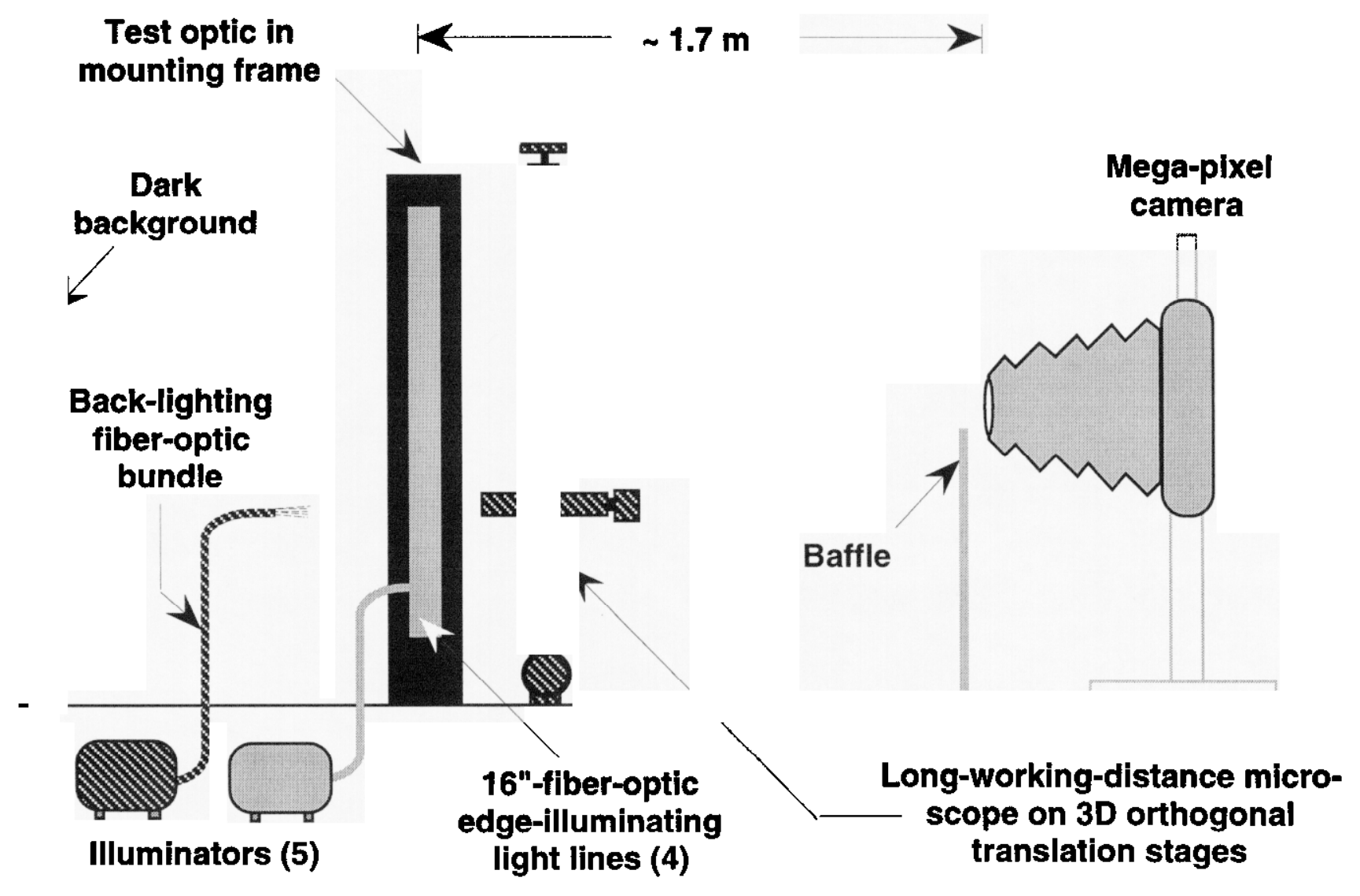

Fig. 1 Schematic of the damage-mapping components. The black element is the optic in a mounting frame. The gray elements are the mapping system. The cross-hatched elements represent the detailed microscopy system.

The first component is shown in black in the figure. The frame holds a unique optic with only minor edge obscuration. It can also hold up to four linear, fiber-optic, light lines from the second component (shown in gray) around the edges of the optic. The light lines (typically $40.6-\mathrm{cm}$ long) provide relatively uniform, white-light illumination from fiber-optic light sources through the edges of the optic into the bulk material. Although the light diverges within the optic, it will encounter total internal reflection at the optic faces. This guarantees at least one pass of white light from each edge across the optic until it strikes the opposite edge. Bulk defects within the uniformly illuminated volume scatter some of this light which is readily visible by eye and to photographic imaging. Note that surface defects such as scratches and pits are effectively bright bulk scatter sites since their damage extends beneath the substrate surfaces. A mega-pixel, digital camera is used to capture an image of the optic with the highlighted bulk scatter sites. We selected a linear-array camera which scans across the object to generate a computer image with dimensions as great as $7520 \times 6000$ pixels. This image is manipulated by computer to produce an accurately aligned and sized image. Each defect can then be ascribed with a unique two-dimensional address on the optic. This address is used by the third component (shown cross-hatched) to position a long-working-distance microscope with two translation stages at the defect site. The third dimension is determined by translating the third stage until a microscope is focused on the defect within the bulk material.

\section{MAPPING PROCEDURES}

\subsection{Generating a map}

A damage map is generated in four steps. First a rapid, low-level exposure (about one minute in duration) is made of the entire optic including edges. The edges and corners typically produce much more scatter than the bulk 
defects so they are much brighter. With just enough intensity to expose these edges, the rotational alignment and exact sizing of the image can be defined and later be adjusted by computer.

Next, a much longer exposure (as long as $\mathbf{2 0}$ minutes) is made of the optic with the saturating edge and corner illumination blocked. Some of the brighter defects may be saturated, but even faintly scattering defects can be detected. The primary emphasis is to obtain the location of the defects rather than their relative scattering levels. Such an image is shown in Fig. 2a.

A negative of this image is then created for convenience. This is rotated to the proper orientation and resized to provide an image of the exact dimensions of the optic. This is accomplished with the information obtained in the first step. A border is drawn in to define the optic edge (see Fig. $2 b$ ). In this particular case the border represents the edges of a $39-\mathrm{cm}$ square focus lens. A coordinate origin is established to define the location of artifacts in two dimensions.

Finally, under higher computer magnification, the image is scanned and marked for the location of all obvious defects (see Fig. 2c). The diamonds highlight the approximate locations of defects. Only the regions defined by rectangles and circles in this particular lens had been exposed to laser irradiation, and hence laser damage. All other artifacts were pre-existing bulk or surface defects.

\subsection{Detailed microscopy}

Individual artifacts can now be examined under highresolution using a long-working-distance microscope. The working distance must be great enough to allow focusing throughout the entire thickness of the optic. Fig. 3 shows the process whereby an artifact is located and photographed.

From the map generated in the fourth step, the $x$ - and $y$ coordinates are obtained for a given artifact. The translation stages for the microscope are positioned at the same $x-y$ coordinates and the microscope is then focused into the bulk material on the artifact under examination. The z-coordinate is then defined at best focus. Since the map image was generated with the mega-pixel camera at a finite distance from the object (typically $1.7 \mathrm{~m}$ ), some parallax will occur with the $x$-y location of defects depending on how deep they were within the bulk

Fig. 2 Successive steps in generating a damage map include (a) an original image, (b) the negative of the image properly rotated, sized and defined with edge borders, and (c) the identification of artifacts under higher magnification.
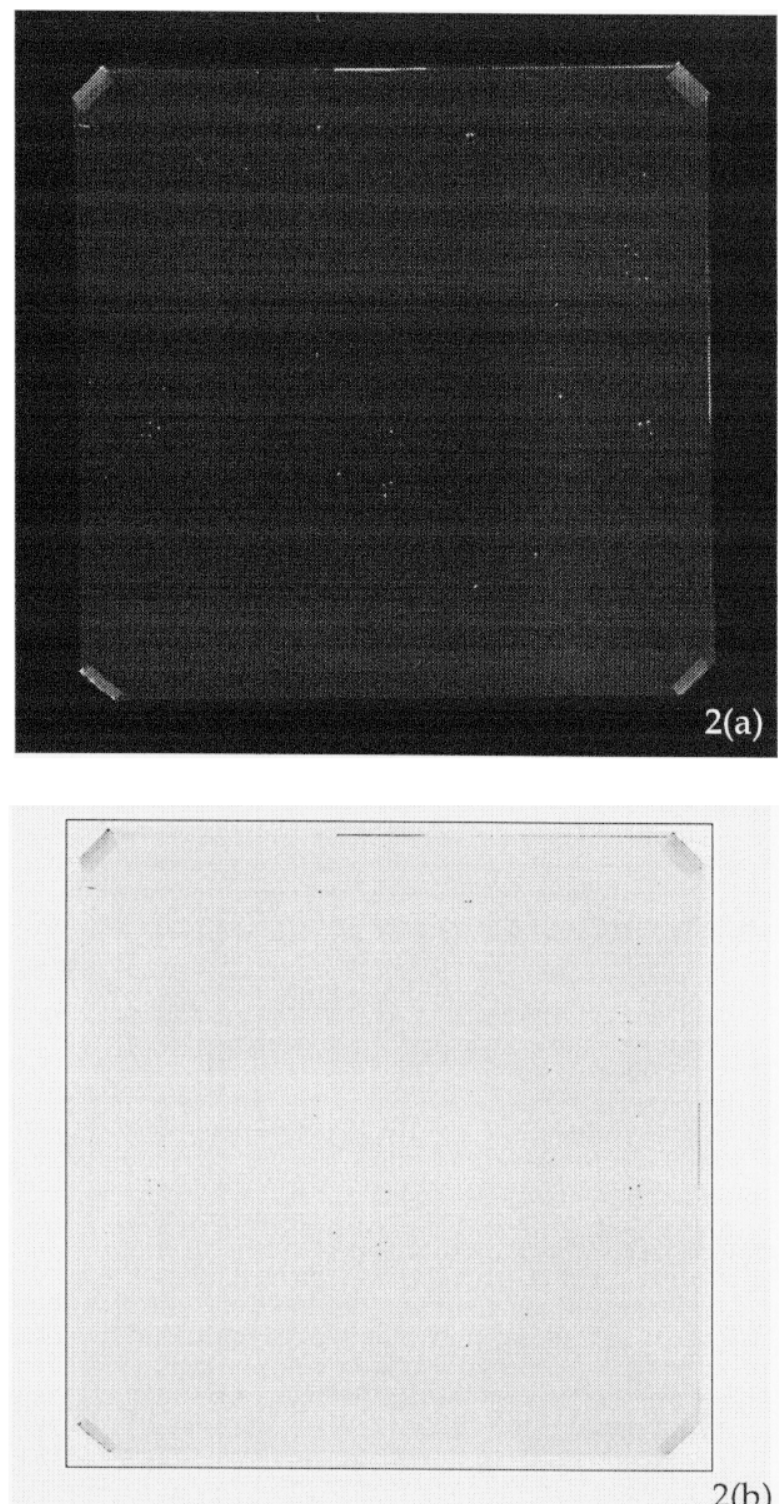

2(b)

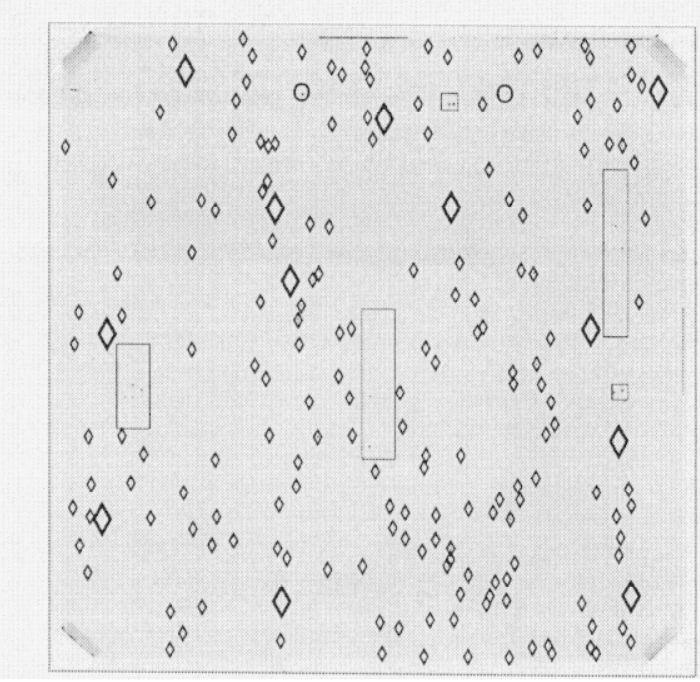

2(c) 
of the substrate. This is, however, not great enough to prevent the artifact from being located. Back illumination with another fiber-optic bundle provides the best detailed images although the edge-illuminating light lines help provide some contrast. As noted earlier, relative intensities of scattered light are not indications of artifact size. Typically more light will be scattered into the microscope from artifacts in the bulk or near the rear surface of the substrate than near the front surface. The degree of light scatter also depends on the morphology of the artifact pits, scratches, bubbles, inclusions or laser-induced damage.

\section{EXAMPLES OF ARTIFACT MORPHOLOGIES}

Fig. 4 shows a reduced version of a full-sized plot of the focus lens previously shown in Figs. 2 and 3. Surrounding it are examples of artifact and damage morphologies. The small, low-resolution images are displayed in greater detail in Figs. 6-9. All are shown at the same magnification as the scale in Fig. 5. Besides an identifying number and brief descriptor, each image is also coded with an F, R or B to signify that the artifact was respectively on the front (incident) surface, rear (exit) surface or in the bulk of the substrate. Images with a black border are sites that have been enhanced with laser irradiation of varying fluences at $355 \mathrm{~nm}$. Those without borders show preexisting artifacts.

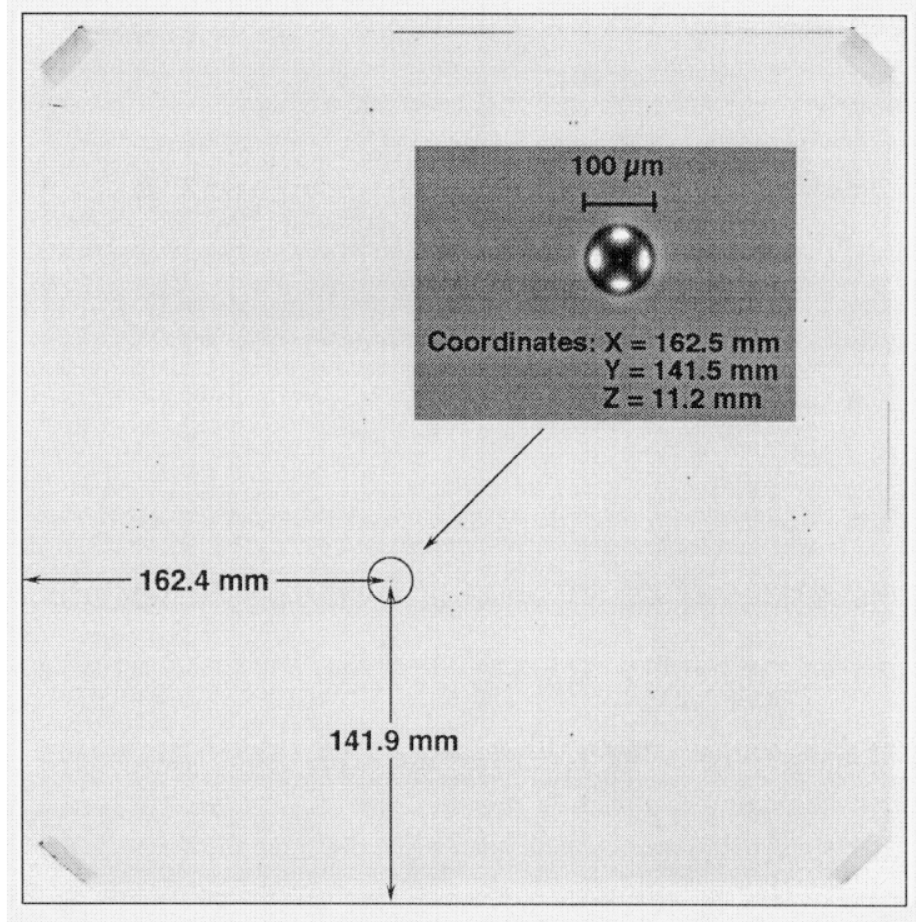

Fig. 3 Coordinates generated in the mapping stage for an artifact (in this case a bubble in the bulk of the substrate) agree well with the coordinates for the focused object observed with the microscope (inset).

Beginning with the image at the top of Fig. 4 and proceeding clockwise the detailed images are representative of the following types of morphologies:

\section{Top of Fig. 4}

Fig. 6(a): a large $400-\mu \mathrm{m}$ piece of lint on the rear surface

6(b): a $40-\mu \mathrm{m}$ pre-existing pit on the front surface

$6(\mathrm{c}): 400-\mu \mathrm{m}$-sized massive damage on the rear surface after numerous shots at successively increasing fluences at $355 \mathrm{~nm}$

$6(\mathrm{~d}): 350-\mu \mathrm{m}$ front-surface contamination, presumably saliva 


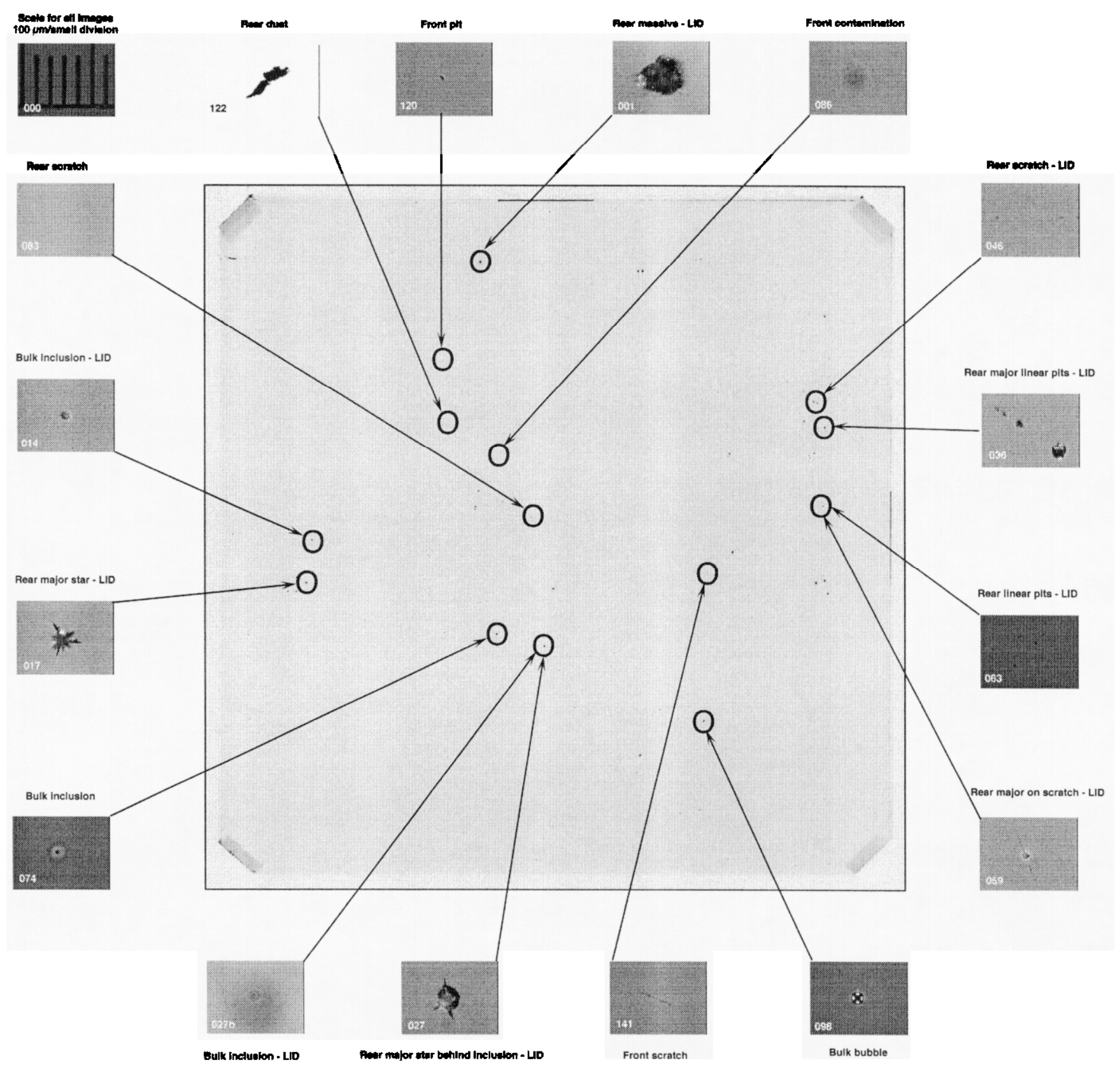

Fig. 4 A reduced version of a full-sized plot of a $39-\mathrm{cm}$ focus lens. The surrounding images are examples of artifact morphologies. They are shown in greater detail in Figs. 6-9.

Fig. 5 Scale for all images shown in Figs. 6 - 9.

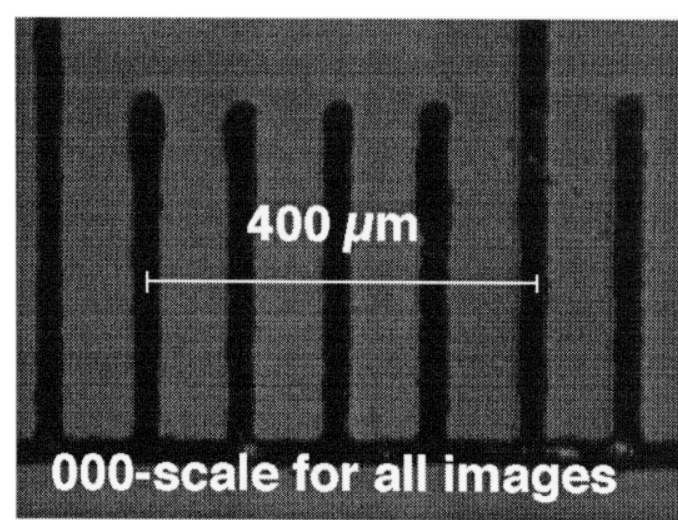


6(a)

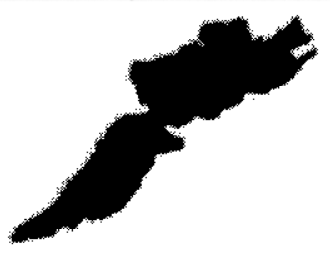

122-R-dust

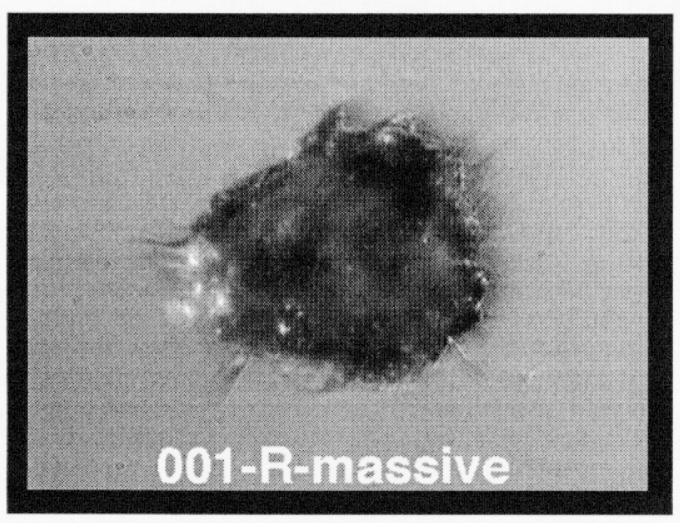

6(c)
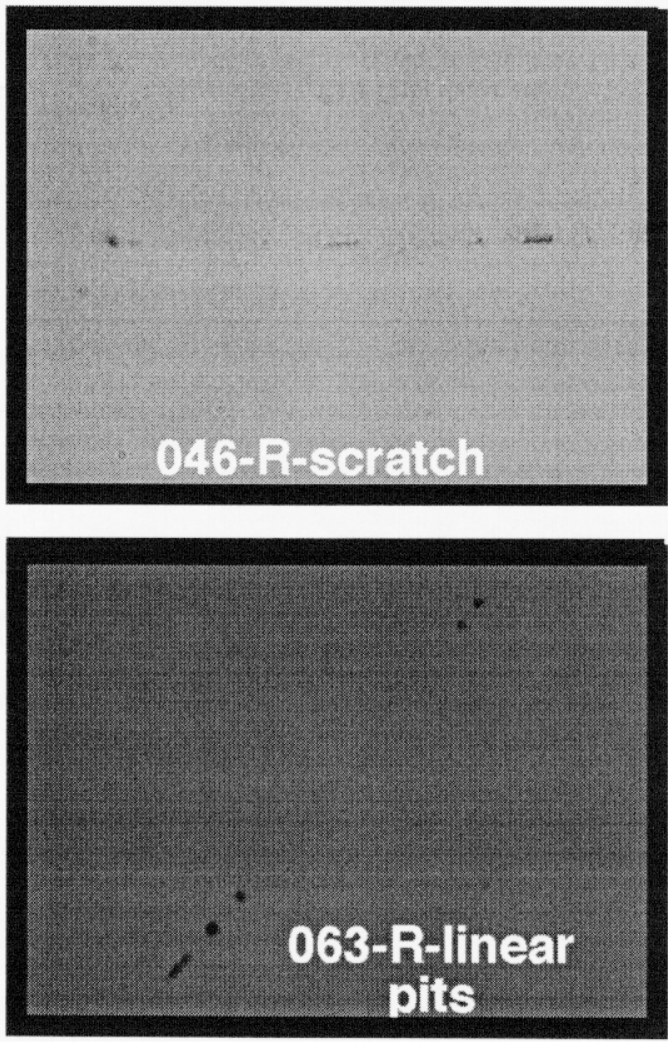

6(b)

Fig. 6

Detailed images from the top of Fig. 4. A

laser-irradiated site is shown with a

black border.

\section{0-F-pit}

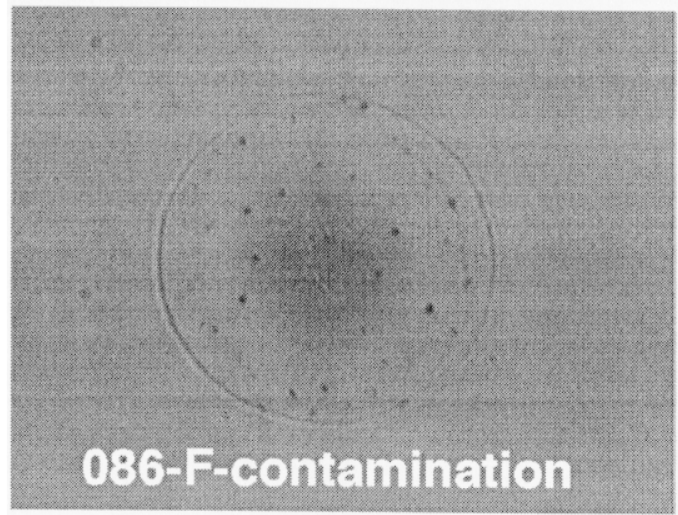

\section{$6(d)$}

086-F-contamination
Fig. 7

Detailed images from the right side of Fig. 4 . Laser-irradiated sites are shown with a black border.
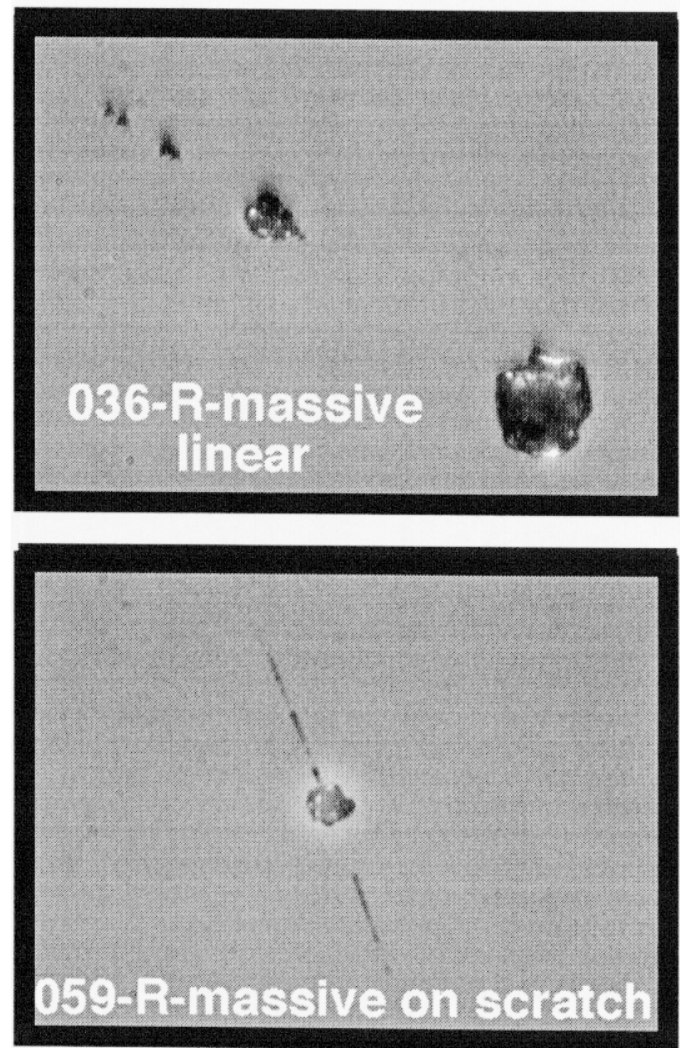

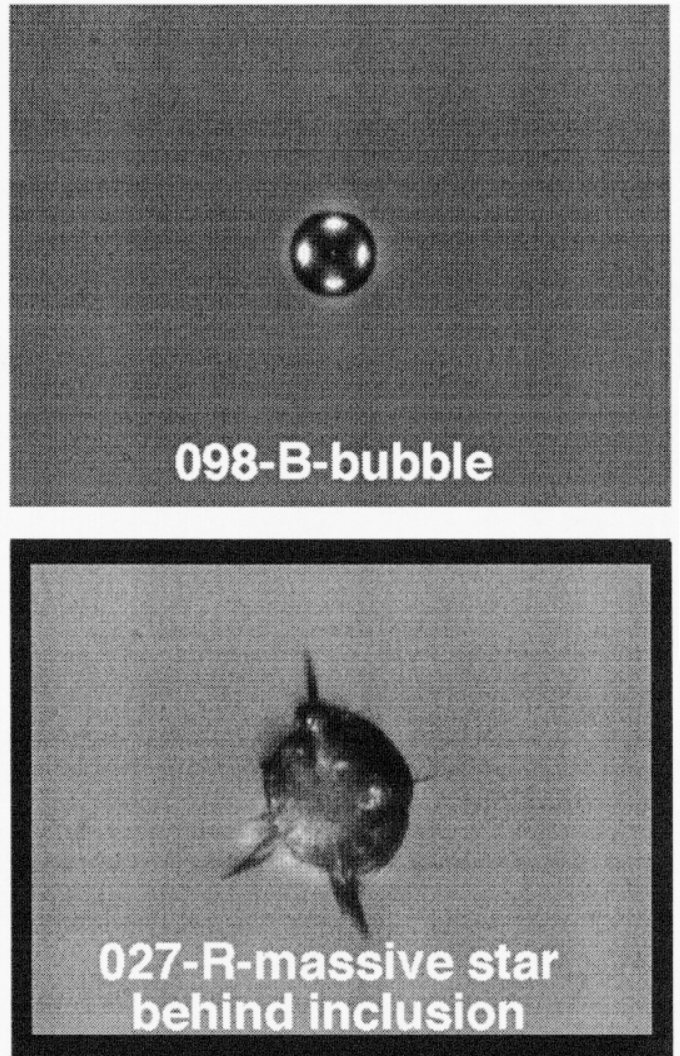

8(c)
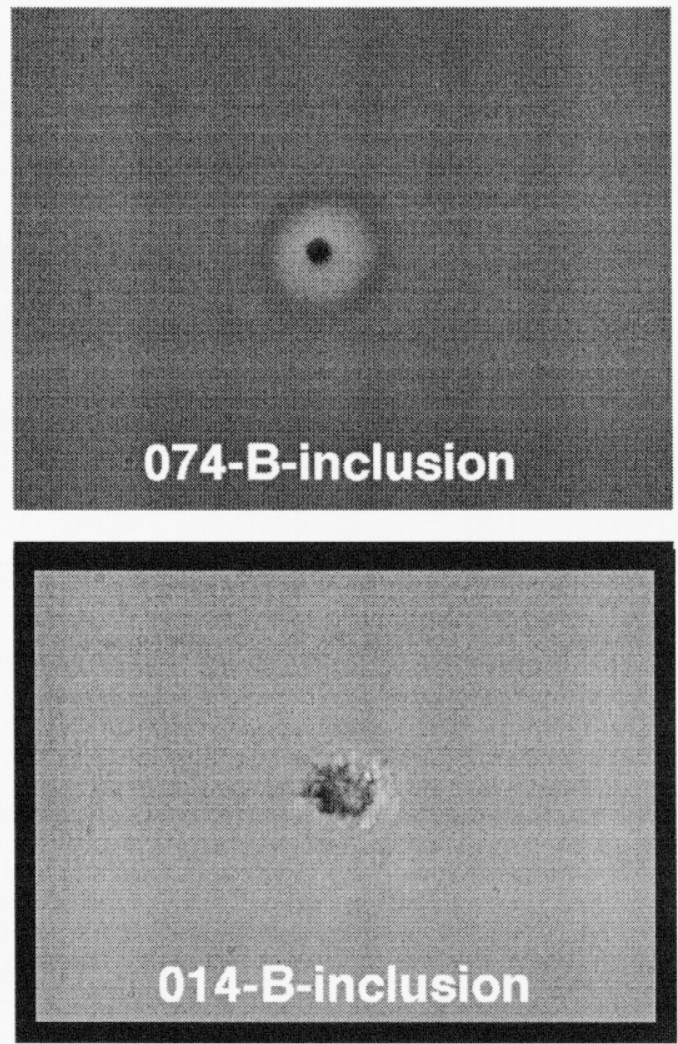

$8(\mathbf{a})$ 8(b)

Fig. 8

Detailed images from the bottom of Fig. 4 .

Laser-irradiated sites are shown with a black border.

Fig. 9

Detailed images from the left side of Fig. 4. Laser-irradiated sites are shown with a black border.

9(c) 8(d)

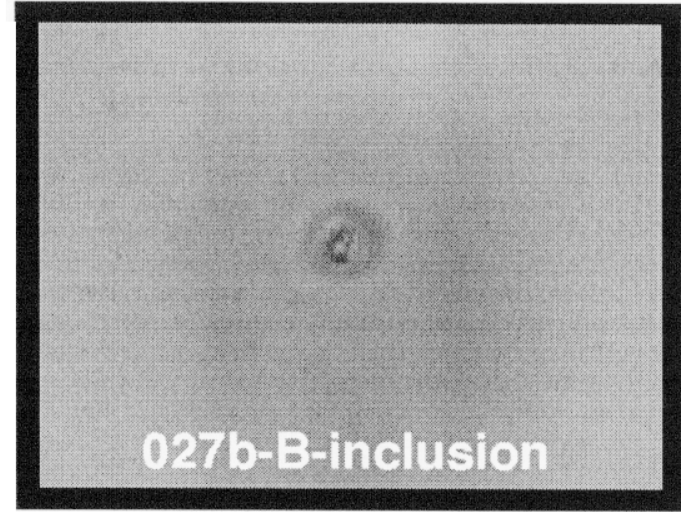

9(d)

\section{1-F-scratch}

$$
\text { 9(b) }
$$
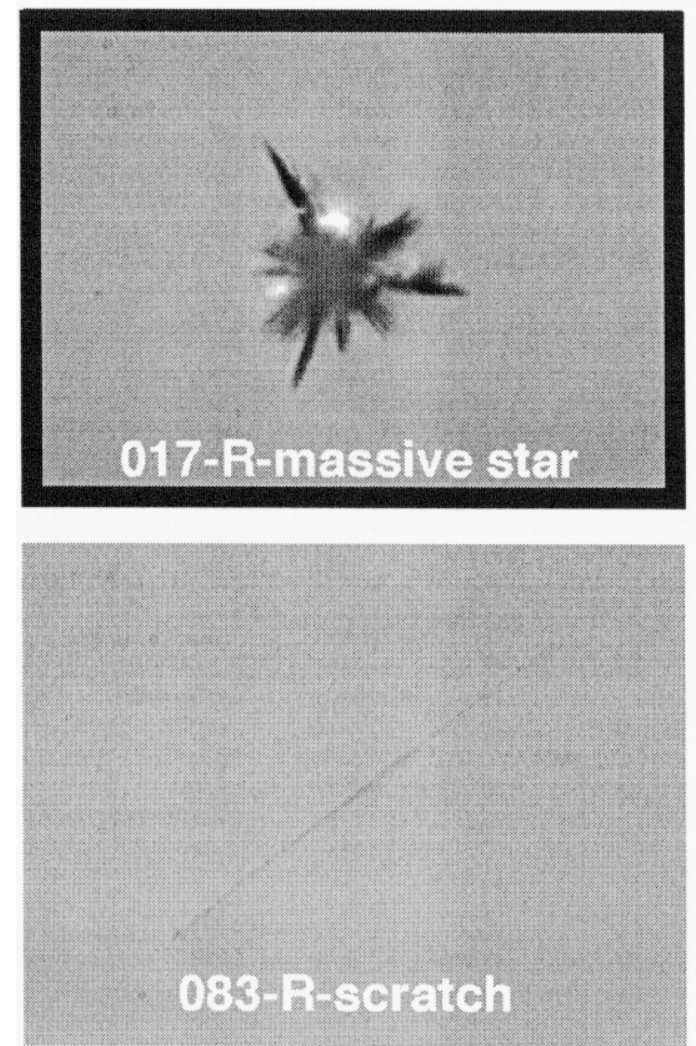


\section{Right side of Fig. 4}

Fig. 7(a): a rear-surface scratch highlighted by a single laser shot per site

$7(b)$ : massive rear-surface pits (up to $130-\mu \mathrm{m}$ in size) in a straight line (without otherwise evidence of a scratch) after single laser shots

$7(\mathrm{c})$ : rear surface pits arrayed in a line after single laser shots

$7(\mathrm{~d}): 60-\mu \mathrm{m}$ massive damage on a rear-surface scratch after single laser shots

\section{Bottom of Fig. 4}

Fig. 8(a): a 100- $\mu$ m unirradiated bubble in the bulk material (bubbles typically will not gnerate damage with laser irradiations and their images show the distinct reflections of the four edge-illuminating light lines)

8 (b): part of a longer unirradiated front-surface scratch

8(c): massive, rear-surface, star-shaped laser damage $(320 \mu \mathrm{m})$ behind the damaged bulk inclusion shown in Fig. 8(d) after single laser shots

8(d): single-shot bulk laser damage $(40 \mu \mathrm{m})$ of an inclusion with a $100-\mu \mathrm{m}$ halo (situated a few $\mathrm{mm}$ in front of, and presumably the source of, the rear-surface damage of Fig. 8(c))

\section{Left side of Fig. 4}

Fig. 9(a): a 30- $\mu \mathrm{m}$ unirradiated bulk inclusion with a $130-\mu \mathrm{m}$ halo

9(b): massive, rear-surface star damage $(300 \mu \mathrm{m})$ after single shot irradiation

9(c): a $120-\mu \mathrm{m}$ bulk inclusion damaged with single laser shots

9(d): part of an unirradiated, rear-surface scratch

\section{EXAMPLES OF MAPPING APPLICATIONS}

We have applied variations of the damage mapping technology to a variety of applications which are described below.

\subsection{Surface scratch quantification}

As noted earlier, surface scratches are efficiently detected by the mapping system because they extend into the bulk material. Typically scratches are on the order of one or more tens of $\mu \mathrm{m}$ wide. We have calibrated our system with independent sources and are in good agreement when measuring a 10$\mu \mathrm{m}$ wide scratch. Fig. 10 shows a well defined scratch 5 - $\mu \mathrm{m}$ wide. Smaller scratches have been detected but their widths have not been resolved.

\subsection{Superposition of damage from successive optics}

Diffraction from a damage site in one

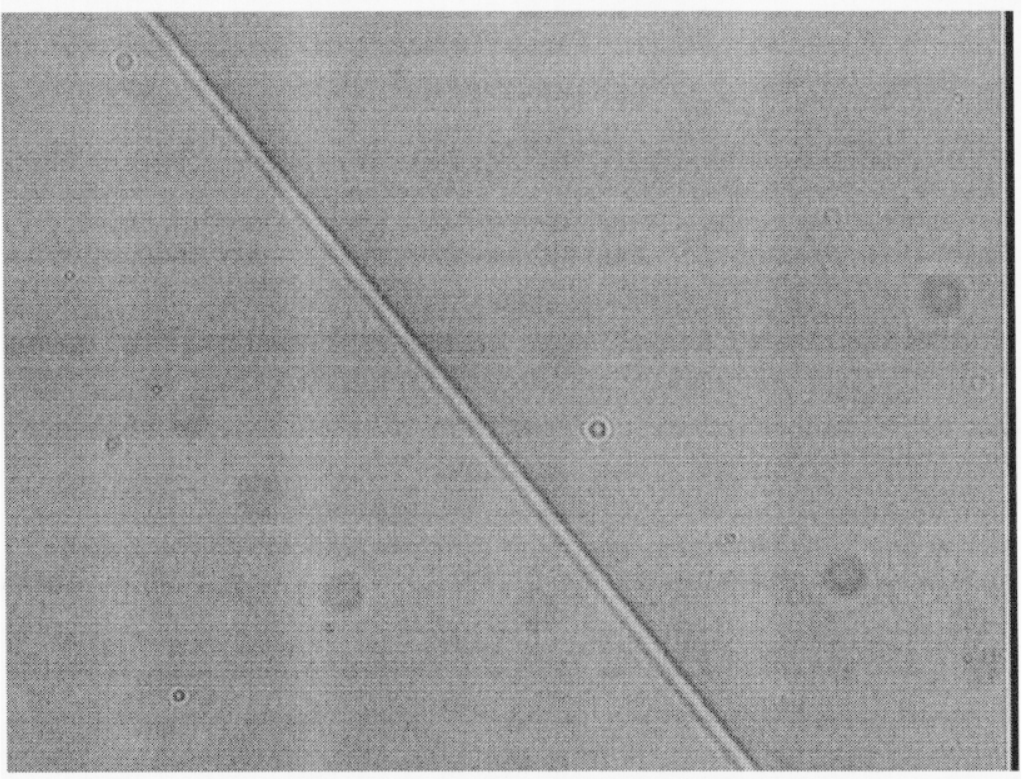

Fig. 10 Aclean 5- $\mu$ m-wide scratch can readily be detected and resolved by the damage mapping system. optic in a laser chain can lead to damage in downstream optics if the beam modulation gets excessive. Fig. 11 shows the superposition of damage sites from three large optics removed from the Beamlet laser. These consisted of a KDP doubling crystal, followed after a few $\mathrm{cm}$ by a KD*P tripling crystal, with a final focus lens about $1 \mathrm{~m}$ away. Although these optics were subjected to, and damaged by, high-fluence laser shots at 1053, 527 and 351-nm, no damage sites ever coincidedat the same $x-y$ coordinates between the three optics. The closest superposition was several $\mathrm{mm}$ apart. The mapping system allowed for very accurate comparison of the locations of damage in each of the three optics. 


\subsection{Monitoring of surface cleanliness}

Surface artifacts can be highlighted uniformly if the fiber-optic light lines illuminate an optic at grazing incidence near the front or rear surfaces of the optic rather than through the bulk of the substrate via its edges. Fig. 12 shows a 12-inch-square substrate of highly contaminated fused silica which has deliberately not been cleaned. Only two light lines were used to begin a scan of the bulk material at the right side. Half way through the scan an additional light line was held in front of the left side of the optic at grazing incidence. The remaining scan displayed the high concentration of surface contamination. Only very slight surface contamination was visible on the right half due to bulk light scattering off the ground-surface edges of the substrate.

\subsection{Edge-clad flaw detection}

Large neodymium-doped laser slabs require absorptive edge claddings around their perimeters to suppress amplified spontaneous emission. Defects such as pits, scratches, dirt or fingerprints that are present when the edge cladding is attached to the laser slabs can cause the cladding to fail and delaminate under high

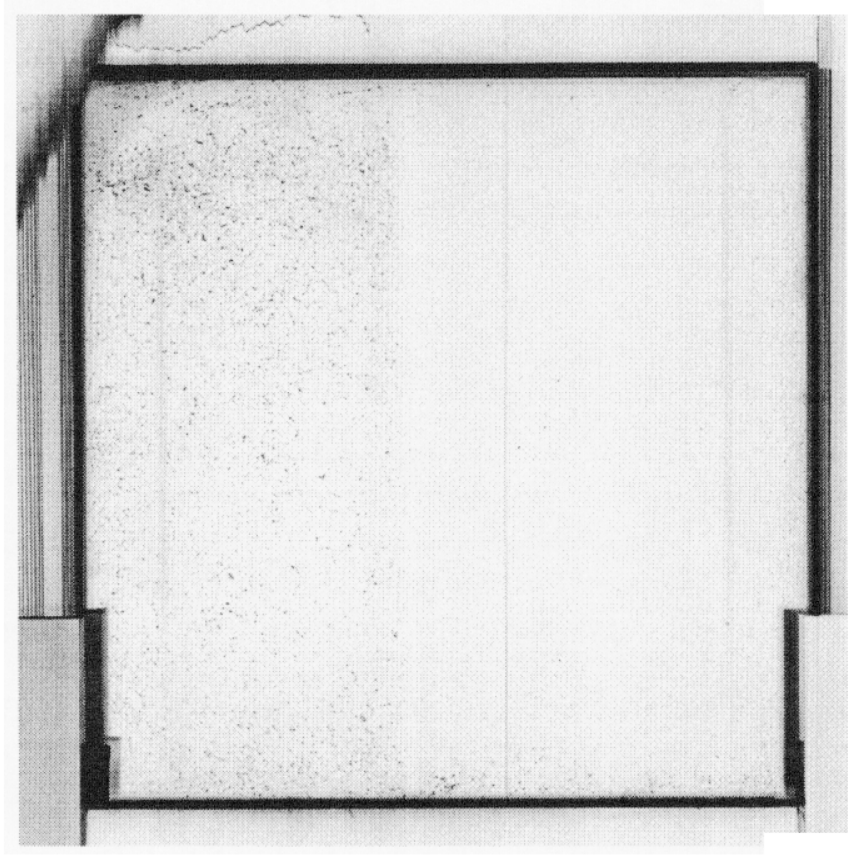

Fig. 12 The right half of this slab of very contaminated fused silica was scanned only with edge illumination. The left half was also illuminated from the front at grazing incidence which highlighted all of the loose surface debris.

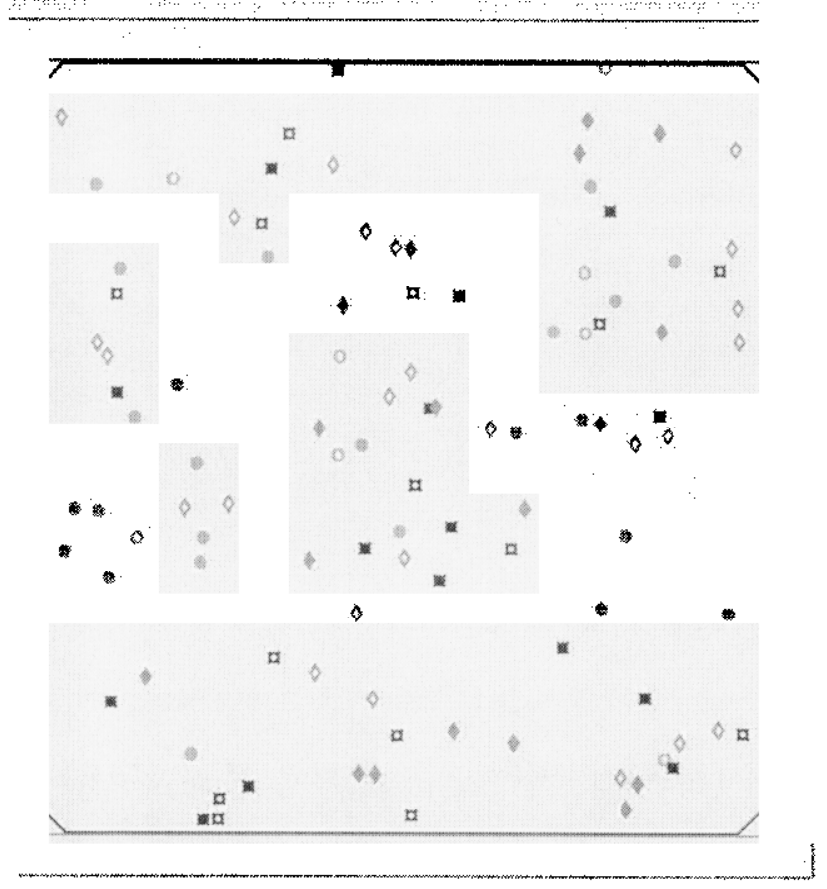

Fig. 11 The superposition of damage sites from three sequential optics removed from the Beamlet laser showed no damage to coincide from the KDP doubling crystal ( $\square$ ), the $\mathrm{KD}^{*} \mathrm{P}$ tripling crystal $(\diamond)$, and the final focus lens $(0)$.

temperature loading from flashlamps. Fig. 13 shows a poorly reproduced image of scratches that were detected via the light-line illumination with edge illumination. Defectively applied claddings can thus be detected and reapplied before they might fail in actual laser use.

\subsection{Laser-slab platinum inclusion inspection}

Platinum inclusions in laser slabs can result in massive damage to the glass under high-fluence laser irradiation. Therefore, before the final finishing of the slabs, they are irradiated at low fluences to highlight potential defects. The inspection of the slabs after this irradiation is a tedious and time-consuming process using high intensity, hot light sources. We have employed a linear light-line configuration to uniformly illuminate entire laser slabs at one time to provide for ease of inspection of platinum-induced damage. Fig. 14 shows a drawing of this configuration. Because of the high absorption of white light within the slab and edge cladding we have employed a two-layer arrangement of light lines to provide sufficient bulk illumination. Four light lines are positioned at each long edge and two at each short edge. The technique is so efficient that it has now been employed as the standard for laser slab inspection. 


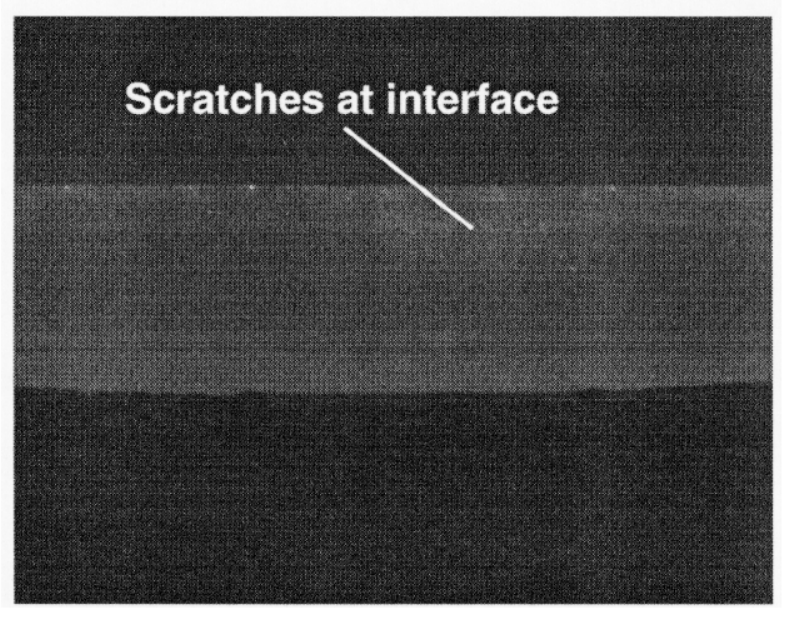

Fig. 13 Linear light lines provide uniform illumination to highlight flaws in laser-slab edge cladding.

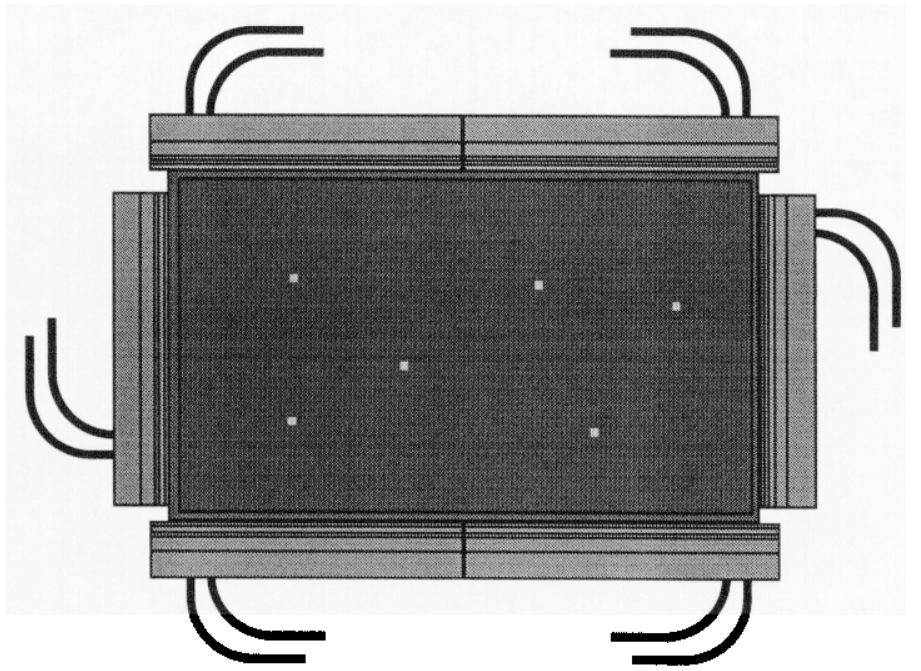

Fig. 14 Linear light lines are now being used to efficiently illuminate entire laser slabs for platinum inclusions and laser-induced damage.

\section{CONCLUSIONS}

We have successfully demonstrated a cost-effective means of diagnosing both damage and damage-generating artifacts in large-scale optics employed in large laser systems. Artifacts can be resolved and diagnosed to dimensions $\leq 5 \mu \mathrm{m}$. The large-area damage mapping system has already been used to detect potentially fatal bulk inclusions and surface scratches which would essentially have been undetected until high-fluence laser irradiations had produced major damage growing to dimensions approaching $1 \mathrm{~mm}$. The system has been employed to diagnose artifacts and damage in fused-silica focusing lenses, debris shields, diffraction gratings, and vacuum windows, in addition to other materials such as large frequency-conversion crystals and slabs of laser glass. The system, or a subset of it, has already been or will be replicated for further inspection and diagnostic use at LLNL as well as other laboratories and commercial establishments, in particular with optical fabrication, processing and testing vendors. An immediate benefit has been the use of linear light lines to detect damage due to platinum inclusions in laser glass.

Although the system is not limited by the number of defects it can detect in optics ranging up to $1 \mathrm{~m}$ in size, it becomes a very time consuming process to diagnose them manually when their number exceed 100. Efforts are currently under way at LLNL to automate the operations by improved computer-controlled search and diagnostic techniques. Also, as originally conceived, a more generic fixturing method needs to be fabricated to support both a variety of different-sized optics, including round ones, and also illuminating fiber-optic sources such as linear light lines and circular cross-section bundles. All of this must be accomplished under class 100 to 1000 clean room conditions.

\section{ACKNOWLEDGMENTS}

The author wishes to acknowledge the advice and help of Derek Decker at LLNL who first implemented an on-line edge-illuminated diagnostic to monitor the evolution of damage of large optics on the Beamlet laser system. Thanks are also extended to Jack Campbell for suggesting the project and providing supervisorial support; to Bill Steele, Ed Lindsey and Joseph Martinez for the early digital camera evaluations; to Stephen Maricle and Hannan Orrell for many of the later measurements; to numerous individuals involved in the fixturing design and procurement; to the clean-room staff; and to the laser-damage-measurement staff.

This work was performed under the auspices of the U. S. Department of Energy by Lawrence Livermore National Laboratory under Contract No. W-7405-ENG-48. 


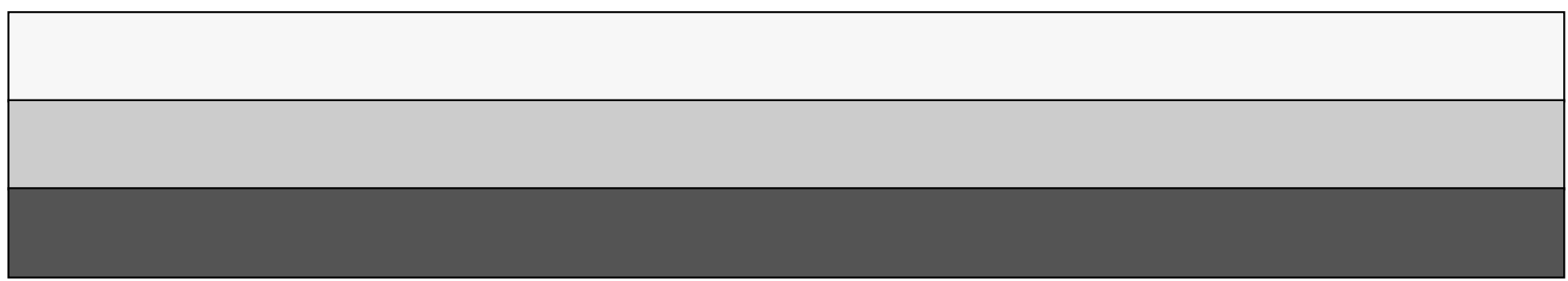

